\title{
LIPSCHITZ STABILITY OF IMPULSIVE FUNCTIONAL-DIFFERENTIAL EQUATIONS
}

\author{
D. D. BAINOV' ${ }^{1}$ and I. M. STAMOVA ${ }^{2}$
}

(Received 2 June 1997; revised 10 April 2000)

\begin{abstract}
An initial value problem is considered for impulsive functional-differential equations. The impulses occur at fixed moments of time. Sufficient conditions are found for Lipschitz stability of the zero solution of these equations. An application in impulsive population dynamics is also discussed.
\end{abstract}

\section{Introduction}

Functional-differential equations are adequate mathematical models of various real processes and phenomena and hence the fundamental and qualitative theory of these equations has been the subject of intensive study in recent years $([4,5,7-9,11,12])$.

Impulsive functional-differential equations are natural generalizations of functional-differential equations. In spite of the great possibilities for application, the mathematical theory of these equations is developing rather slowly due to technical and theoretical difficulties which are connected with the specific features of these equations $([1,2])$.

In the present paper we define the notion of Lipschitz stability of solutions of a system of impulsive functional-differential equations. Let us note that this notion has been introduced by Dannan and Elaydi [6] for ordinary differential equations without impulses.

By virtue of a comparison equation and differential inequalities for piecewise continuous functions, sufficient conditions are found for Lipschitz stability of the zero solution of a system of nonlinear impulsive functional-differential equations.

\footnotetext{
${ }^{1}$ Medical University of Sofia, P.O. Box 45, Sofia 1504, Bulgaria.

${ }^{2}$ Technical University, Sliven, Bulgaria.

(C) Australian Mathematical Society 2001, Serial-fee code 0334-2700/01
} 


\section{Preliminary notes and definitions}

Let $\mathbb{R}^{n}$ be the $n$-dimensional Euclidean space with the norm $|\cdot|, \mathbb{R}_{+}=[0, \infty)$, $t_{0} \in \mathbb{R}$ and $\tau>0$.

We consider the system of impulsive functional-differential equations

$$
\begin{aligned}
\dot{x}(t) & =f\left(t, x(t), x_{t}\right), & & t>t_{0}, t \neq t_{k}, \\
\Delta x\left(t_{k}\right) & =x\left(t_{k}+0\right)-x\left(t_{k}-0\right)=I_{k}\left(x\left(t_{k}-0\right)\right), & t_{k} & >t_{0}, k=1,2, \ldots,
\end{aligned}
$$

where $f:\left(t_{0}, \infty\right) \times \mathbb{R}^{n} \times D \rightarrow \mathbb{R}^{n} ; D=\left\{\Phi:[-\tau, 0] \rightarrow \mathbb{R}^{n}, \Phi(t)\right.$ is continuous everywhere except a finite number of points $\tilde{t}$ at which $\Phi(\tilde{t}-0)$ and $\Phi(\tilde{t}+0)$ exist and $\Phi(\tilde{t}-0)=\Phi(\tilde{t})\} ; I_{k}: \mathbb{R}^{n} \rightarrow \mathbb{R}^{n}, k=1,2, \ldots ; t_{0}<t_{1}<t_{2}<\cdots ; \lim _{k \rightarrow \infty} t_{k}=\infty$ and for $t>t_{0}, x_{t} \in D$ is defined by $x_{t}(s)=x(t+s),-\tau \leq s \leq 0$.

Let $\varphi_{0} \in D$. We denote by $x(t)=x\left(t ; t_{0}, \varphi_{0}\right)$ the solution of the system (1), which satisfies the initial conditions

$$
\begin{aligned}
& x\left(t ; t_{0}, \varphi_{0}\right)=\varphi_{0}\left(t-t_{0}\right), \quad t_{0}-\tau \leq t \leq t_{0}, \\
& x\left(t_{0}+0 ; t_{0}, \varphi_{0}\right)=\varphi_{0}(0) .
\end{aligned}
$$

The solution $x\left(t ; t_{0}, \varphi_{0}\right)=x(t)$ of the initial value problem (1) and (2) is characterized by the following properties.

(a) For $t_{0}-\tau \leq t \leq t_{0}$ the solution $x(t)$ satisfies the initial conditions (2).

(b) For $t_{0}<t \leq t_{1}$ the solution $x(t)=x\left(t ; t_{0}, \varphi_{0}\right)$ of the initial value problem (1) and (2) coincides with the solution of the problem

$$
\begin{aligned}
\dot{x}(t) & =f\left(t, x(t), x_{t}\right), \quad t>t_{0}, \\
x_{t_{0}} & =\varphi_{0} .
\end{aligned}
$$

At the moment $t=t_{1}$ the mapping point $\left(t, x\left(t ; t_{0}, \varphi_{0}\right)\right)$ moves 'instantly' from the position $\left(t_{1}, x\left(t_{1} ; t_{0}, \varphi_{0}\right)\right)$ into the position $\left(t_{1}, x\left(t_{1} ; t_{0}, \varphi_{0}\right)+l_{1}\left(x\left(t_{1} ; t_{0}, \varphi_{0}\right)\right)\right)$.

(c) For $t_{1}<t \leq t_{2}$ the solution of (1) and (2) coincides with the solution of the problem

where

$$
\begin{aligned}
\dot{y}(t) & =f\left(t, y(t), y_{t}\right), \quad t>t_{1}, \\
y_{t_{1}} & =\varphi_{1}, \quad \varphi_{1} \in D,
\end{aligned}
$$

$$
\varphi_{1}\left(t-t_{1}\right)= \begin{cases}\varphi_{0}\left(t-t_{1}\right), & t \in\left[t_{0}-\tau, t_{0}\right] \cap\left[t_{1}-\tau, t_{1}\right], \\ x\left(t ; t_{0}, \varphi_{0}\right), & t \in\left(t_{0}, t_{1}\right), \\ x\left(t ; t_{0}, \varphi_{0}\right)+I_{1}\left(x\left(t ; t_{0}, \varphi_{0}\right)\right), & t=t_{1} .\end{cases}
$$

At the moment $t=t_{2}$ the mapping point $\left(t, x\left(t ; t_{0}, \varphi_{0}\right)\right)$ moves 'instantly' and so on. 
The solution $x(t)=x\left(t ; t_{0}, \varphi_{0}\right)$ of the initial value problem (1) and (2) is a piecewise continuous function for $t>t_{0}$ with points of discontinuity of the first kind $t_{k}, k=$ $1,2, \ldots$, where it is continuous from the left.

We also introduce the notation:

$$
\begin{gathered}
I=\left[t_{0}-\tau, \infty\right), \quad I_{0}=\left[t_{0}, \infty\right), \\
G_{k}=\left\{(t, x) \in I_{0} \times \mathbb{R}^{n}: t_{k-1}<t<t_{k}\right\}, \quad k=1,2, \ldots, \quad \text { and } \quad G=\bigcup_{k=1}^{\infty} G_{k} .
\end{gathered}
$$

Let $J \subset \mathbb{R}$ be an interval. We define the following classes of functions:

$P C\left[J, \mathbb{R}^{n}\right]=\left\{\sigma: J \rightarrow \mathbb{R}^{n}: \sigma(t)\right.$ is continuous everywhere except some points $t_{k}$ at which $\sigma\left(t_{k}-0\right)$ and $\sigma\left(t_{k}+0\right)$ exist and $\left.\sigma\left(t_{k}-0\right)=\sigma\left(t_{k}\right)\right\}$;

$P C^{1}\left[J, \mathbb{R}^{n}\right]=\left\{\sigma \in P C\left[J, \mathbb{R}^{n}\right]: \sigma(t)\right.$ is continuously differentiable everywhere except some points $t_{k}$ at which $\dot{\sigma}\left(t_{k}-0\right)$ and $\dot{\sigma}\left(t_{k}+0\right)$ exist and $\left.\dot{\sigma}\left(t_{k}-0\right)=\dot{\sigma}\left(t_{k}\right)\right\}$

$\mathscr{K}=\left\{a \in C\left[\mathbb{R}_{+}, \mathbb{R}_{+}\right]: a(u)\right.$ is a strictly increasing function and $\left.a(0)=0\right\} ;$ $\mathscr{V}_{0}=\left\{V: I_{0} \times \mathbb{R}^{n} \rightarrow \mathbb{R}_{+}: V \in C\left[G, \mathbb{R}_{+}\right], V\right.$ satisfies locally a Lipschitz condition with respect to $x \in \mathbb{R}^{n}$ on each of the sets $G_{k}$, $V\left(t_{k}-0, x\right)=V\left(t_{k}, x\right)$ and $V\left(t_{k}+0, x\right)=\lim _{\substack{t>t_{k} \\ t \rightarrow t_{k}}} V(t, x)$ exists $\}$ $\Omega_{1}=\left\{x \in P C\left[I_{0}, \mathbb{R}^{n}\right]: V(s, x(s)) \leq V(t, x(t)), t \stackrel{t \rightarrow t_{k}}{\leq} \leq s \leq t, t \geq t_{0}\right.$, $\left.V \in \mathscr{V}_{0}\right\}$

Let $V \in \mathscr{V}_{0}, x \in P C\left[I_{0}, \mathbb{R}^{n}\right]$ and $t \neq t_{k}, k=1,2, \ldots$

We introduce the function

$$
D_{-} V(t, x(t))=\liminf _{h \rightarrow 0^{-}} \frac{1}{h}\left[V\left(t+h, x(t)+h f\left(t, x(t), x_{t}\right)\right)-V(t, x(t))\right] .
$$

We consider the system (1) together with

$$
\begin{array}{ll}
\dot{u}=g(t, u), & t \neq t_{k}, t>t_{0}, \\
u\left(t_{k}+0\right)=B_{k}\left(u\left(t_{k}\right)\right), & t_{k}>t_{0}, k=1,2, \ldots,
\end{array}
$$

where $g: I_{0} \times \mathbb{R}_{+} \rightarrow \mathbb{R}_{+}, B_{k}: \mathbb{R}_{+} \rightarrow \mathbb{R}_{+}, k=1,2, \ldots$

We denote by $u^{+}\left(t ; t_{0}, u_{0}\right)$ the maximal solution of (3), for which $u^{+}\left(t_{0}+0 ; t_{0}, u_{0}\right)=$ $u_{0} \in \mathbb{R}_{+}$.

Let $\rho>0$. In what follows we will use the notation:

$$
\begin{aligned}
B_{\rho} & =\left\{x \in \mathbb{R}^{n}:|x|<\rho\right\} ; \\
\left\|\varphi_{0}\right\| & =\sup _{s \in[-r, 0]}\left|\varphi_{0}(s)\right|, \quad \varphi_{0} \in D ; \quad \text { and } \\
\|A\|_{1} & =\sup _{|x|=1}|A x| \text { is the norm of the } n \times n \text {-matrix } A .
\end{aligned}
$$


Now we shall define two kinds of Lipschitz stability for the zero solution of the system (1).

DEFINITION 1. The zero solution of the system (1) is said to be:

(a) uniformly Lipschitz stable if

$$
(\exists M>0)(\exists \delta>0)\left(\forall \varphi_{0} \in D:\left\|\varphi_{0}\right\|<\delta\right)\left(\forall t \in I_{0}\right):\left|x\left(t ; t_{0}, \varphi_{0}\right)\right| \leq M\left\|\varphi_{0}\right\| ;
$$

(b) globally uniformly Lipschitz stable if

$$
(\exists M>0)\left(\forall \varphi_{0} \in D\right)\left(\forall t \in I_{0}\right):\left|x\left(t ; t_{0}, \varphi_{0}\right)\right| \leq M\left\|\varphi_{0}\right\|
$$

When studying Lipschitz stability of the zero solution of the system (1) we will use the following definitions of Lipschitz stability of the zero solution of (3) ([10]).

DEFINITION 2. The zero solution of (3) is said to be:

(a) uniformly Lipschitz stable if

$$
(\exists M>0)(\exists \delta>0)\left(\forall u_{0} \in \mathbb{R}_{+}: u_{0}<\delta\right)\left(\forall t>t_{0}\right): u^{+}\left(t ; t_{0}, u_{0}\right) \leq M u_{0} ;
$$

(b) globally uniformly Lipschitz stable if

$$
(\exists M>0)\left(\forall u_{0} \in \mathbb{R}_{+}\right)\left(\forall t>t_{0}\right): u^{+}\left(t ; t_{0}, u_{0}\right) \leq M u_{0}
$$

We introduce the following conditions.

H1. $t_{0}<t_{1}<t_{2}<\cdots$.

H2. $\lim _{k \rightarrow \infty} t_{k}=\infty$.

H3. $f \in P C\left[I_{0} \times \mathbb{R}^{n} \times D, \mathbb{R}^{n}\right]$.

H4. $f(t, 0,0)=0, t \in I_{0}$.

H5. $I_{k} \in C\left[\mathbb{R}^{n}, \mathbb{R}^{n}\right], k=1,2, \ldots$

H6. $I_{k}(0)=0, k=1,2, \ldots$.

H7. $g \in P C\left[I_{0} \times \mathbb{R}_{+}, \mathbb{R}\right]$.

H8. $g(t, 0)=0, t \in I_{0}$.

H9. $B_{k} \in \mathscr{K}$ and $B_{k}:\left[0, \rho_{0}\right) \rightarrow[0, \rho), k=1,2, \ldots, \rho_{0}=$ const $>0, \rho=$ const $>0$. H10. For $x \in B_{\rho}$ and for all $k=1,2, \ldots$ the inequalities $\left|x+I_{k}(x)\right| \leq B_{k}(|x|)$ hold. H11. The zero solution of (3) is uniformly Lipschitz stable (globally uniformly Lipschitz stable).

When proving the main results we shall use the following lemmas:

LEMMA 1. Let the following conditions hold.

1. Conditions $\mathrm{H} 1-\mathrm{H} 10$ are met. 
2. The solution $x(t)=x\left(t ; t_{0}, \varphi_{0}\right)$ of the initial value problem (1) and (2) is such that $x \in P C\left[I, B_{\rho}\right] \cap P C^{1}\left[I_{0}, B_{\rho}\right], \rho=$ const $>0$.

3. The function $V \in \mathscr{V}_{0}, V: I_{0} \times B_{\rho} \rightarrow \mathbb{R}_{+}$is such that

$$
\begin{aligned}
& V\left(t_{0}+0, \varphi_{0}(0)\right) \leq u_{0}, \\
& D_{-} V(t, x(t)) \leq g(t, V(t, x(t))), \quad t \in I_{0}, t \neq t_{k}, x \in \Omega_{1}, \\
& V\left(t_{k}+0, x\left(t_{k}\right)+I_{k}\left(x\left(t_{k}\right)\right)\right) \leq B_{k}\left(V\left(t_{k}, x\left(t_{k}\right)\right)\right), \quad k=1,2, \ldots .
\end{aligned}
$$

Then

$$
V\left(t, x\left(t ; t_{0}, \varphi_{0}\right)\right) \leq u^{+}\left(t ; t_{0}, u_{0}\right), \quad t \in I_{0}
$$

PROOF. For $t \in I_{0}$ the maximal solution $u^{+}\left(t ; t_{0}, u_{0}\right)$ of (3) is defined by the equality

$$
u^{+}\left(t ; t_{0}, u_{0}\right)=\left\{\begin{array}{l}
r_{0}\left(t ; t_{0}, u_{0}^{+}\right), \quad t_{0}<t \leq t_{1}, \\
r_{1}\left(t ; t_{1}, u_{1}^{+}\right), \quad t_{1}<t \leq t_{2}, \\
\ldots \ldots \ldots \cdots \cdots \cdots, \\
r_{k}\left(t ; t_{k}, u_{k}^{+}\right), \quad t_{k}<t \leq t_{k+1}, \\
\ldots \ldots \ldots \ldots \cdots \cdots
\end{array}\right.
$$

where $r_{k}\left(t ; t_{k}, u_{k}^{+}\right)$is the maximal solution of the equation without impulses, $\dot{u}=$ $g(t, u)$ in the interval $\left(t_{k}, t_{k+1}\right]$ for which $u_{k}^{+}=B_{k}\left(r_{k-1}\left(t_{k} ; t_{k-1}, u_{k-1}^{+}\right)\right), k=1,2, \ldots$ and $u_{0}^{+}=u_{0}$.

Let $t \in\left(t_{0}, t_{1}\right] \cap I_{0}$. Then the corresponding comparison lemma for the continuous case ([11]) implies

$$
V\left(t, x\left(t ; t_{0}, \varphi_{0}\right)\right) \leq u^{+}\left(t ; t_{0}, u_{0}\right),
$$

that is, the inequality (5) is fulfilled for $t \in\left(t_{0}, t_{1}\right] \cap I_{0}$.

Suppose that (5) holds true for $t \in\left(t_{k-1}, t_{k}\right] \cap I_{0}, k>1$. Then, using (4) and the fact that the function $B_{k}$ is increasing, we obtain

$$
\begin{aligned}
V\left(t_{k}+0, x\left(t_{k}+0 ; t_{0}, \varphi_{0}\right)\right) & \leq B_{k}\left(V\left(t_{k}, x\left(t_{k} ; t_{0}, \varphi_{0}\right)\right)\right. \\
& \leq B_{k}\left(u^{+}\left(t_{k} ; t_{0}, u_{0}\right)\right)=B_{k}\left(r_{k-1}\left(t_{k} ; t_{k-1}, u_{k-1}^{+}\right)\right)=u_{k}^{+} .
\end{aligned}
$$

We apply again the corresponding comparison lemma for functional-differential equations without impulses [11] in the interval $\left(t_{k}, t_{k+1}\right] \cap I_{0}$ and obtain

$$
V\left(t, x\left(t ; t_{0}, \varphi_{0}\right)\right) \leq r_{k}\left(t ; t_{k}, u_{k}^{+}\right)=u^{+}\left(t ; t_{0}, u_{0}\right),
$$

that is, the inequality (5) is true for $t \in\left(t_{k}, t_{k+1}\right] \cap I_{0}$.

The proof is completed by induction. 
LEMMA 2 ([3]). Let the following conditions hold:

1. The functions $u, k \in P C\left[I_{0}, \mathbb{R}_{+}\right]$.

2. $c_{0}=$ const $>0, \beta_{k}=$ const $\geq 0, k=1,2, \ldots$.

3. The function $p \in C\left[\mathbb{R}_{+}, \mathbb{R}_{+}\right]$, $p$ is nondecreasing in $\mathbb{R}_{+}$and positive in $(0, \infty)$.

4. For $t \in I_{0}$ the inequality

$$
u(t) \leq c_{0}+\int_{t_{0}}^{t} k(s) p(u(s)) d s+\sum_{t_{0}<t_{k}<t} \beta_{k} u\left(t_{k}\right)
$$

holds true.

5. $B_{k}=\int_{c_{k}}^{u}(1 / p(s)) d s, k=1,2, \ldots$, where $c_{k}=\left(1+\beta_{k}\right) B_{k-1}^{-1}\left(\int_{t_{k-1}}^{t_{k}} k(s) d s\right)$.

6. $B_{0}=\int_{u_{0}}^{u}(1 / p(s)) d s, u \geq u_{0}>0$.

Then

$$
u(t) \leq B_{k}^{-1}\left(\int_{t_{k}}^{t} k(s) d s\right), \quad t_{k-1}<t \leq t_{k}, \quad k=1,2, \ldots
$$

\section{Main results}

THEOREM 1. Let the following conditions hold:

1. Conditions $\mathrm{H} 1-\mathrm{H} 11$ are valid.

2. For $t \in I_{0}, x \in \Omega_{1} \cap B_{\rho}$ and for sufficiently small $h>0$ the inequality

$$
\left|x(t)+h f\left(t, x(t), x_{t}\right)\right| \leq|x(t)|+h g(t,|x(t)|)+\varepsilon(h)
$$

holds true, where $\varepsilon(h) / h \rightarrow 0$ as $h \rightarrow 0^{-}, t \neq t_{k}, k=1,2, \ldots$

Then the zero solution of the system (1) is uniformly Lipschitz stable (globally uniformly Lipschitz stable).

PROOF, Let $\rho^{*}=\min \left(\rho, \rho_{0}\right)$. It follows from condition $\mathrm{H} 11$ that there exist constants $M>0$ and $\delta>0\left(M \delta<\rho^{*}\right)$ such that for $0 \leq u_{0}<\delta$ and $t>t_{0}$ we have

$$
u^{+}\left(t ; t_{0}, u_{0}\right) \leq M u_{0} .
$$

We will prove that $\left|x\left(t ; t_{0}, \varphi_{0}\right)\right| \leq M\left\|\varphi_{0}\right\|$ for $\left\|\varphi_{0}\right\|<\delta$ and $t>t_{0}$.

Let us suppose the opposite. Then there exists a solution $x(t)=x\left(t ; t_{0}, \varphi_{0}\right)$ of the system (1), $\varphi_{0} \in D:\left\|\varphi_{0}\right\|<\delta$ and $t^{*} \in\left(t_{k}, t_{k+1}\right]$ for some positive integer $k$, such that

$$
\left|x\left(t^{*}\right)\right|>M\left\|\varphi_{0}\right\|, \quad|x(t)| \leq M\left\|\varphi_{0}\right\|, \quad t_{0}<t \leq t_{k} .
$$


It follows from condition $\mathrm{H} 10$ that

$$
\begin{aligned}
\left|x\left(t_{k}+0\right)\right| & =\left|x\left(t_{k}\right)+I_{k}\left(x\left(t_{k}\right)\right)\right| \leq B_{k}\left(\left|x\left(t_{k}\right)\right|\right) \\
& \leq B_{k}\left(M\left\|\varphi_{0}\right\|\right) \leq B_{k}(M \delta) \leq G_{k}\left(\rho^{*}\right) \leq \rho .
\end{aligned}
$$

The last inequality asserts that there exists $t^{0}, t_{k}<t^{0} \leq t^{*}$, such that

$$
M\left\|\varphi_{0}\right\|<\left|x\left(t^{0}\right)\right|<\rho \quad \text { and } \quad|x(t)|<\rho, \quad t<t \leq t^{0} .
$$

Let us define $V(t, x(t))=|x(t)|$ and $u_{0}=\left\|\varphi_{0}\right\|$. Since condition 2 of Theorem 1 is fulfilled, then for $t \in\left(t_{0}, t^{0}\right], t \neq t_{j}, j=1,2, \ldots, k$, the inequalities

$$
\begin{aligned}
D_{-} V(t, x(t)) & =\liminf _{h \rightarrow 0^{-}} \frac{1}{h}[|x(t+h)|-|x(t)|] \\
& \leq \lim _{h \rightarrow 0^{-}} \frac{1}{h}\left[|x(t+h)|+h g(t,|x(t)|)+\varepsilon(h)-\left|x(t)+h f\left(t, x(t), x_{t}\right)\right|\right] \\
& \leq g(t,|x(t)|)+\lim _{h \rightarrow 0^{-}} \frac{\varepsilon(h)}{h}+\lim _{h \rightarrow 0^{-}}\left|\frac{1}{h}[x(t+h)-x(t)]-f\left(t, x(t), x_{t}\right)\right| \\
& =g(t,|x(t)|)=g(t, V(t, x(t)))
\end{aligned}
$$

hold true.

The inequalities

$$
\begin{aligned}
V\left(t_{j}+0, x\left(t_{j}+0\right)\right) & =\left|x\left(t_{j}+0\right)\right|=\left|x\left(t_{j}\right)+I_{j}\left(x\left(t_{j}\right)\right)\right| \\
& \leq B_{j}\left(\left|x\left(t_{j}\right)\right|\right)=B_{j}\left(V\left(t_{j}, x\left(t_{j}\right)\right)\right)
\end{aligned}
$$

follow from condition $\mathrm{H} 10$ for $j=1,2, \ldots, k$.

Since

$$
V\left(t_{0}+0, \varphi_{0}(0)\right)=\left|\varphi_{0}(0)\right| \leq\left\|\varphi_{0}\right\|=u_{0},
$$

it follows from Lemma 1 that

$$
|x(t)|=V(t, x(t)) \leq u^{+}\left(t ; t_{0}, u_{0}\right), \quad t_{0}<t \leq t^{0} .
$$

Hence (6)-(8) imply the inequalities

$$
M\left\|\varphi_{0}\right\|<\left|x\left(t^{0}\right)\right|=V\left(t^{0}, x\left(t^{0}\right)\right) \leq u^{+}\left(t^{0} ; t_{0}, u_{0}\right) \leq M u_{0}=M\left\|\varphi_{0}\right\| .
$$

The contradiction obtained shows that

$$
x\left(t ; t_{0}, \varphi_{0}\right) \mid \leq M\left\|\varphi_{0}\right\| \text { for }\left\|\varphi_{0}\right\|<\delta \text { and } t>t_{0} .
$$


THEOREM 2. Let the following conditions hold:

1. Conditions $\mathrm{H} 1-\mathrm{H} 11$ are met.

2. For $t \in I_{0}$ and $x \in \Omega_{1} \cap B_{\rho}$ the inequality

$$
\left[x(t), f\left(t, x(t), x_{t}\right)\right]_{-} \leq g(t,|x(t)|), \quad t \neq t_{k}, k=1,2, \ldots,
$$

holds true, where

$$
[x, y]_{-}=\liminf _{h \rightarrow 0^{-}} \frac{1}{h}[|x+h y|-|x|], \quad x, y \in \mathbb{R}^{n} .
$$

Then the zero solution of the system (1) is uniformly Lipschitz stable (globally uniformly Lipschitz stable).

The proof of Theorem 2 is analogous to the proof of Theorem 1.

Let us introduce the following conditions:

H12. The function $p \in C\left[\mathbb{R}_{+}, \mathbb{R}_{+}\right], p$ is nondecreasing in $\mathbb{R}_{+}$, positive in $(0, \infty)$ and it is submultiplicative, that is, $p(\lambda u) \leq p(\lambda) p(u)$ for $\lambda>0, u>0$.

H13. $p(\lambda u) \geq \mu(\lambda) p(u)$ for $\lambda>0, u>0$ where $\mu(\lambda)>0$ for $\lambda>0$.

H14. For $\left(t, x, x_{t}\right) \in I_{0} \times \mathbb{R}^{n} \times D,\left|f\left(t, x, x_{t}\right)\right| \leq m(t) p(|x|)$, where $m \in C\left[I_{0}, \mathbb{R}_{+}\right]$. H15. For $x \in \mathbb{R}^{n}$ and $k=1,2, \ldots,\left|I_{k}(x)\right| \leq \beta_{k}|x|, \beta_{k}=$ const $>0$.

THEOREM 3. Let the following conditions hold:

1. Conditions $\mathrm{H} 1-\mathrm{H} 6$ and $\mathrm{H} 12-\mathrm{H} 15$ are met.

2. $\quad B_{k}=\int_{c_{k}}^{u} \frac{d s}{p(s)}, \quad c_{k}=\left(1+\beta_{k}\right) B_{k-1}^{-1}\left(\int_{t_{k-1}}^{t_{k}} m(s) d s\right) ; \quad k=1,2, \ldots \quad$ and

3. $B_{k}(\infty)=\infty, k=0,1,2, \ldots$.

$$
B_{0}=\int_{c}^{u} \frac{d s}{p(s)}, \quad u \geq c>0 .
$$

4. For every $k=0,1,2, \ldots, t \in\left(t_{k}, t_{k+1}\right]$ and $\varphi_{0} \in D$,

$$
B_{k}^{-1}\left(\frac{p\left(\left\|\varphi_{0}\right\|\right)}{\left\|\varphi_{0}\right\|} \int_{t_{k}}^{t} m(s) d s\right) \leq M, \quad 0<M=\text { const }
$$

Then the zero solution of the system (1) is globally uniformly Lipschitz stable.

ProOF. For $t_{k}<t \leq t_{k+1}, k=0,1,2, \ldots$, the function $x(t)=x\left(t ; t_{0}, \varphi_{0}\right)$ satisfies the integral equation

$$
x(t)=x\left(t_{k}\right)+I_{k}\left(x\left(t_{k}\right)\right)+\int_{t_{k}}^{t} f\left(s, x(s), x_{s}\right) d s .
$$


We obtain by induction

$$
x(t)=x\left(t_{0}+0\right)+\sum_{t_{0}<t_{k}<t} I_{k}\left(x\left(t_{k}\right)\right)+\int_{t_{0}}^{t} f\left(s, x(s), x_{s}\right) d s, \quad t>t_{0} .
$$

It then follows from conditions $\mathrm{H} 12-\mathrm{H} 15$ that

$$
\begin{aligned}
\left|x\left(t ; t_{0}, \varphi_{0}\right)\right| & \leq\left|\varphi_{0}(0)\right|+\sum_{t_{0}<t_{k}<t}\left|I_{k}\left(x\left(t_{k}\right)\right)\right|+\int_{t_{0}}^{t}\left|f\left(s, x(s), x_{s}\right)\right| d s \\
& \leq\left\|\varphi_{0}\right\|+\sum_{t_{0}<t_{k}<t} \beta_{k}\left|x\left(t_{k}\right)\right|+\int_{t_{0}}^{t} m(s) p(|x(s)|) d s,
\end{aligned}
$$

whence we obtain the estimates, for $t>t_{0}$,

$$
\begin{aligned}
\frac{\left|x\left(t ; t_{0}, \varphi_{0}\right)\right|}{\left\|\varphi_{0}\right\|} & \leq 1+\sum_{t_{0}<t_{k}<t} \beta_{k} \frac{\left|x\left(t_{k} ; t_{0}, \varphi_{0}\right)\right|}{\left\|\varphi_{0}\right\|}+\int_{t_{0}}^{t} \frac{m(s)}{\left\|\varphi_{0}\right\|} p\left(\left\|\varphi_{0}\right\| \frac{\left|x\left(s ; t_{0}, \varphi_{0}\right)\right|}{\left\|\varphi_{0}\right\|}\right) d s \\
& \leq 1+\int_{t_{0}}^{t} \frac{p\left(\left\|\varphi_{0}\right\|\right)}{\left\|\varphi_{0}\right\|} m(s) p\left(\frac{\left|x\left(s ; t_{0}, \varphi_{0}\right)\right|}{\left\|\varphi_{0}\right\|}\right) d s+\sum_{t_{0}<t_{k}<t} \beta_{k} \frac{\left|x\left(t_{k} ; t_{0}, \varphi_{0}\right)\right|}{\left\|\varphi_{0}\right\|} .
\end{aligned}
$$

We apply Lemma 2 to the last inequality and obtain

$$
\left|x\left(t ; t_{0}, \varphi_{0}\right)\right| \leq\left\|\varphi_{0}\right\| B_{k}^{-1}\left(\frac{p\left(\left\|\varphi_{0}\right\|\right)}{\left\|\varphi_{0}\right\|} \int_{t_{0}}^{t} m(s) d s\right),
$$

$t \in\left(t_{k}, t_{k+1}\right], k=0,1,2, \ldots$

The last inequality and condition 4 of Theorem 3 imply $\left|x\left(t ; t_{0}, \varphi_{0}\right)\right| \leq M\left\|\varphi_{0}\right\|$ for all $\varphi_{0} \in D$ and $t>t_{0}$.

\section{Applications in impulsive population dynamics}

We consider the impulsive population model

$$
\begin{aligned}
& \dot{N}(t)=N(t)\left[a+b N(t-\tau)-c N^{2}(t-\tau)\right], \quad t \neq t_{k}, \\
& \Delta N\left(t_{k}\right)=I_{k}\left(N\left(t_{k}\right)\right), \quad t_{k}>0, k=1,2, \ldots,
\end{aligned}
$$

where the velocity $\dot{N}(t)$ of increase in population is a quadratic form of the density $N(t)$ of the same population; $\tau>0$ characterizes the maturity of the population; $a, c \in(0, \infty), b \in \mathbb{R}$ are constants, different for the concrete population; $I_{k}, k=$ $1,2, \ldots$, characterize the value of the increase or decrease of the population under the action of external perturbations (for example, human action) at the moments $0<t_{1}<t_{2}<\cdots$ and $\lim _{k \rightarrow \infty} t_{k}=\infty$. 
THEOREM 4. Let the following conditions hold:

1. $\left[N(t), N(t)\left(a+b N(t-\tau)-c N^{2}(t-\tau)\right)\right]_{-} \leq p(t) F(N(t))$, for $t \geq 0$, $|N(t)|<\rho,|N(t-\tau)|<\rho, F \in \mathscr{K}, p \in C\left[I_{0}, \mathbb{R}_{+}\right]$.

2. $\left|N+I_{k}(N)\right| \leq B_{k}(|N|),|N|<\rho, k=1,2, \ldots$, where $B_{k} \in \mathscr{K}$ and $B_{k}:\left[0, \rho_{0}\right) \rightarrow[0, \rho), k=1,2, \ldots$

3. For each $h \in\left(0, \rho_{0}\right)$,

$$
\int_{t_{k}}^{t_{k+1}} p(s) d s+\int_{h}^{B_{k}(h)} \frac{d s}{F(s)} \leq 0, \quad k=1,2, \ldots .
$$

Then the zero solution of the system (9) is uniformly Lipschitz stable.

PROOF. It follows from the conditions of Theorem 4 that the zero solution of the system

$$
\begin{aligned}
& \dot{N}(t)=p(t) F(N(t)), \quad t \neq t_{k}, \quad t>0, \\
& N\left(t_{k}+0\right)=B_{k}\left(N\left(t_{k}\right)\right), \quad k=1,2, \ldots,
\end{aligned}
$$

is uniformly Lipschitz stable ([10]).

Since all conditions of Theorem 2 are fulfilled, then the zero solution of the system (9) is uniformly Lipschitz stable.

\section{Acknowledgement}

This work was partially supported by the Bulgarian Ministry of Education and Science under Grant MM-702.

\section{References}

[1] D. D. Bainov, V. C. Covachev and I. M. Stamova, "Estimates of the solutions of impulsive quasilinear functional differential equations", Ann. Fac. Sci. de Toulouse 2 (1991) 149-161.

[2] D. D. Bainov, V. C. Covachev and I. M. Stamova, "Stability under:persistent disturbances of impulsive differential-difference equations of neutral type", J. Math. Anal. Appl. 187 (1994) 790808.

[3] D. D. Bainov and P. S. Simeonov, Integral inequalities and applications (Kluwer Academic Publishers, Dordrecht, 1992).

[4] R. Bellman and K. Cooke, Differential-difference equations (Academic Press, London, 1963).

[5] T. A. Burton, Stability and periodic solutions of ordinary and functional differential equations (Academic Press, London, 1985).

[6] F. M. Dannan and S. Elaydi, "Lipschitz stability of nonlinear systems of differential equations", J. Math. Anal. Appl. 113 (1986) 562-577. 
[7] L. E. El'sgol'ts and S. B. Norkin, Introduction to the theory and application of differential equations with deviating arguments (Academic Press, London, 1973).

[8] J. Hale, Theory of functional differential equations (Springer, New York, 1977).

[9] J. Kato, "On Lyapunov-Razumikhin type theorems for functional differential equations", Funkc. Ekv. 16 (1973) 225-239.

[10] G. K. Kulev and D. D. Bainov, "Lipschitz stability of impulsive systems of differential equations", Intern. J. Theor. Phys. 30 (1991) 737-756.

[11] V. Lakshmikantham, S. Leela and A. A. Martynyuk, Stability analysis of nonlinear systems (Marcel Dekker, New York, 1989).

[12] E. Pinney, Ordinary difference-differential equations (University of California Press, Berkeley and Los Angeles, 1958). 